\title{
Cascaded Transforms for Signature Recognition with Image Registration
}

\author{
M. Mani Roja \\ Research Scholar \\ Sant Baba Gadge Amravati University \\ Asso.Prof, TSEC, Mumbai
}

\author{
Sudhir Sawarkar \\ Principal \\ Datta Meghe College of Engineering \\ Mumbai
}

\begin{abstract}
Biometrics methods of human identification have gained much attention recently, mainly because they easily deal with most problems of traditional identification, since users are identified by who they are, not by something they have to remember or carry with them. This paper attempts to improve the performance of signature based authentication system by cascading orthogonal transforms. The signatures are acquired using WACOM digital pen tablet and then features are extracted. Our experimental results on the image data set from 75 users, confirm the advantages of our cascaded approach over unimodal approaches. It should be noted that a distinct advantage of the proposed system is that it does not require multiple signature samples for training.
\end{abstract}

\section{General Terms}

Biometrics, Pattern Recognition, Image Registration

\section{Keywords}

Discrete wavelet transform Discrete Cosine Transform; Discrete Sine Transform

\section{INTRODUCTION}

Many different types of unique physiological or behavioral characteristics exist for humans. Some of the more traditional uses of these biometric methods for identification or verification include $[1,2]$ :

i) Fingerprint recognition: Humans have used fingerprints for personal identification for many centuries. A fingerprint is the pattern of ridges and valleys on the surface of a fingertip. Fingerprints of identical twins are different and so are the prints on each finger of the same person. Fingerprints of a small fraction of the population may be unsuitable for automatic identification because of genetic factors, aging, environmental, or occupational reasons (e.g., manual workers may have a large number of cuts and bruises on their fingerprints that keep changing).Fingerprint recognition systems rely on the biometric device's ability to distinguish the unique impressions of ridges and valleys made by an individual's finger.

ii) Facial recognition: Face recognition is a non-intrusive method. The most popular approaches to face recognition are based on the location and shape of facial attributes, such as the eyes, eyebrows, nose, lips, and chin and their spatial relationships or the global analysis of the face image. In order that a facial recognition system works well in practice, it should automatically detect whether a face is present in the acquired image; locate the face if there is one; and recognize the face from any pose. iii) Signature Recognition: The way a person signs her name is known to be a characteristic of that individual. Although signatures require contact with the writing instrument and an effort on the part of the user, they have been accepted in government, legal, and commercial transactions as a method of verification. Dynamic signature verification not only compares the signature itself, but also marks changes in speed, pressure and timing that occur during signing.

iv) Voice recognition: Voice is a combination of physiological and behavioral biometrics. Voice recognition biometrics digitizes a profile of a person's speech into a template voiceprint and stores it as a table of binary numbers. During authentication, the spoken phrase is compared to the previously stored template. A text-independent voice recognition system recognizes the speaker independent of what she speaks. A text independent system is more difficult to design than a text-dependent system, but offers more protection against fraud. A disadvantage of voice-based recognition is that speech features are sensitive to a number of factors such as background noise.

v) Hand geometry: Hand geometry recognition systems are based on a number of measurements taken from the human hand, including its shape, size of palm, and lengths and widths of the fingers.

vi) Retina scanning: The retinal vasculature is rich in structure and is supposed to be a characteristic of each individual and each eye. It is claimed to be the most secure biometric since it is not easy to change or replicate the retinal vasculature. Retinal scanning involves an electronic scan of the retina, the innermost layer of the wall of the eyeball.

vii) Iris scanning: Iris scanning uses a camera mounted between three and ten feet away from the person to take a high definition photograph of the individual's eyes. The iris is the annular region of the eye bounded by the pupil and the sclera on either side. The complex iris texture carries very distinctive information useful for personal recognition.

viii) Palm print: The palms of the human hands contain pattern of ridges and valleys much like the fingerprints. The area of the palm is much larger than the area of a finger and as a result, palm prints are expected to be even more distinctive than the fingerprints. Since palm print scanners need to capture a large area, they are bulkier and more expensive than the fingerprint sensors.

ix) Keystroke dynamics: Keystroke dynamics technology measures dwell time (the length of time a person holds down each key) as well as flight time (the time it takes to move between keys). Taken over the course of several login 
sessions, these two metrics produce a measurement of rhythm unique to each user.

Other technologies that are emerging or that are being studied include vein patterns, facial thermograph, DNA, sweat pores, hand grip, fingernail bed, body odor, ear shape, gait, skin luminescence, brain wave pattern, footprint recognition and foot dynamics.

\subsection{Need for Signature Recognition System}

Signature verification by computers has received extensive research interest in the field of pattern recognition [3]. As ones signature may change over time and it is not nearly as unique or difficult to forge as iris patterns or fingerprints, however signature's widespread acceptance by the public makes it more suitable for lower authentication needs. Use of signature as an authentication method has already become a tradition in the western civilization and is respected among the others. The signature is an accepted proof of identity of the person in a transaction on his or her authentication method. For many years the problem of signature verification has generally been solved by some authorities or clerical employee, however with the invention of computers and scanning devices the trend has been towards automation of the whole process. The accuracy of offline signature verification system depends upon the robustness of feature vector. As a consequence, more and more researchers have looked into the feature extraction.

\section{BIOMETRIC SYSTEMS}

A biometric system is essentially a pattern recognition system that operates by acquiring biometric data from an individual, extracting a feature set from the acquired data, and comparing this feature set against the template set in the database. Depending on the application, a biometric system may operate either in verification mode or identification mode [4]:

In the verification mode, the system validates a person's identity by comparing the captured biometric data with her own biometric template stored in the system database. In the identification mode, the system recognizes an individual by searching the templates of all the users in the database for a match. Therefore, the system conducts a one-to-many comparison to establish an individual's identity to be.

A simple biometric system has four important modules [5, 6]:

- Sensor module which captures the biometric data of an individual. An example is a fingerprint sensor that captures fingerprint impressions of a user.

- Feature extraction module in which the acquired data is processed to extract feature values. For example, the position and orientation of minutiae points in a fingerprint image would be computed in the feature extraction module of a fingerprint system.

- Matching module in which the feature values are compared against those in the template by generating a matching score. For example, in this module, the number of matching minutiae between the query and the template can be computed and treated as a matching score.

- Decision-making module in which the user's claimed identity is either accepted or rejected based on the matching score generated in the matching module.

\section{PROPOSED SYSTEM}

The block diagram of the proposed method for signature authentication is shown in fig. 1. The signature from every user is acquired using the Wacom Digital pen tablet [7]. After preprocessing, Discrete wavelet Transform (DWT) coefficients are calculated as first level feature vectors. These feature vectors are cascaded with Discrete Sine transform (DST) and Discrete Cosine transform (DCT). The final feature vectors are saved in the database for comparison.

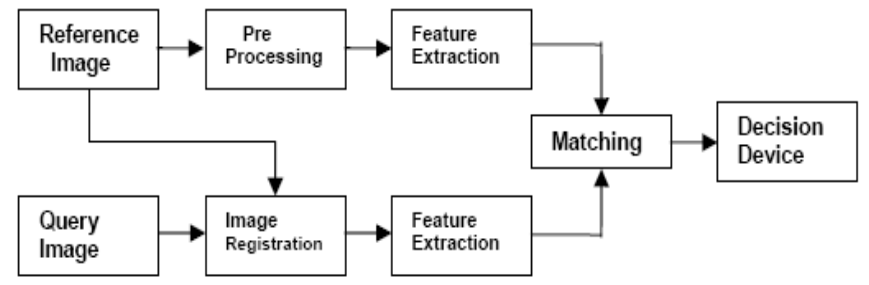

Fig 1: Block diagram of signature recognition system

\subsection{Image Acquisition and Pre-processing}

Our image acquisition is inherently simple and does not employ any special illumination. The WACOM bamboo digital pen tablet as shown in fig. 2 was used to acquire the signatures. Five signature samples from 75 users have been collected. Hence our data base consists of 375 signature samples. These images have different dimensions therefore images are scaled to a dimension of $128 \times 256$. After scaling, the next step is colour normalization and binarization. After this, image becomes ready for feature extraction phase.

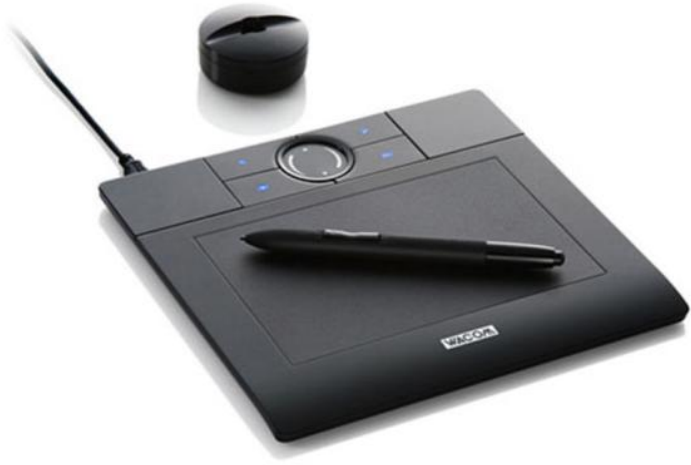

Fig 2: Wacom Digital Pen tablet

\subsection{FEATURE EXTRACTION}

To extract the feature vectors from the signature template, we have used the following transforms.

\subsection{1 .Discrete Wavelet Transform}

The Wavelet transform [8-10] is a transform which provides the time-frequency representation. Wavelet transform is capable of providing the time and frequency information simultaneously, hence giving a time-frequency representation of the signal. In numerical analysis and functional analysis, a discrete wavelet transform (DWT) is any wavelet transform for which the wavelets are discretely sampled. 
Let us define the scaled $\left(\varphi_{j, m, n}(x, y)\right.$ and translated $\psi_{j, m, n}^{i}(x, y)$ basis functions for two dimensional discrete waveform as

$$
\begin{gathered}
\varphi_{j, m, n}(x, y)=2^{\frac{J}{2}} \varphi\left(2^{J} x-m, 2^{J} y-n\right) \\
\psi_{j, m, n}^{i}(x, y)=2^{\frac{J}{2}} \psi\left(2^{J} x-m, 2^{J} y-n\right), i=\{H, V, D\}
\end{gathered}
$$

The discrete wavelet transform of image $\mathrm{f}(\mathrm{x}, \mathrm{y})$ of size $\mathrm{M} \mathrm{x} \mathrm{N}$ is given by

$$
\begin{aligned}
& W_{\varphi}\left(j_{0}, m, n\right)=\frac{1}{\sqrt{M N}} \sum_{x=0}^{M-1} \sum_{y=0}^{N-1} f(x, y) \varphi_{j_{o}, m, n}(x, y) \\
& W_{\psi}^{i}(j, m, n)=\frac{1}{\sqrt{M N}} \sum_{x=0}^{M-1} \sum_{y=0}^{N-1} f(x, y) \psi_{j, m, n}(x, y)
\end{aligned}
$$

where $\mathrm{i}=\{\mathrm{H}, \mathrm{V}, \mathrm{D}\}, \mathrm{j}_{0}$ is an arbitrary starting scale and $W_{\varphi}\left(j_{0}, m, n\right)$ coefficients define the approximation of image $\mathrm{f}(\mathrm{x}, \mathrm{y})$ at scale $\mathrm{j}_{0}$. The $W_{\psi}^{i}(j, m, n)$ coefficients add horizontal, vertical and diagonal details foe scales $\mathrm{j} \geq 0$. Normally $\mathrm{j}_{0}$ is selected as 0 and $\mathrm{N}=\mathrm{M}=2^{\mathrm{J}}$ so that $\mathrm{j}=1,2, . \mathrm{J}$ 1 and $\mathrm{m}=\mathrm{n}=0,1,2 \ldots, .2^{\mathrm{J}}-1$.

The 2D DWT can be implemented using digital filters and down samplers. Fig. 3 shows the process in block diagram form.

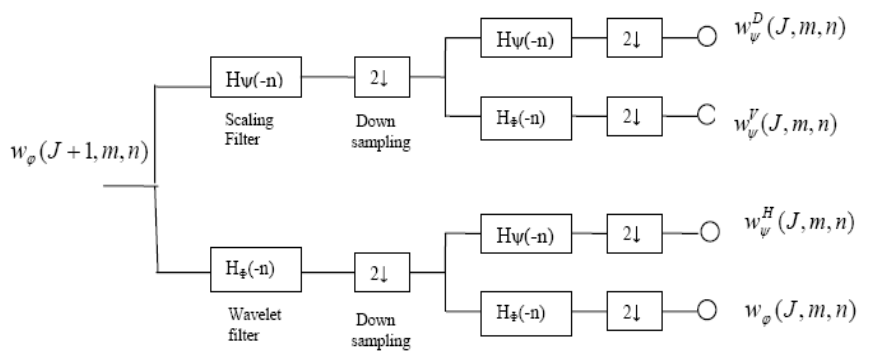

Fig 3: The DWT filtering operations

In fig 4 , image $\mathrm{f}(\mathrm{x}, \mathrm{y})$ is used as the $w_{\varphi}(J+1, m, n)$ input. Convolving its rows with $\mathrm{H}_{\psi}(-n)$ and $\mathrm{H}_{\Phi}(-n)$ and down sampling its column, we get two images whose horizontal resolutions are reduced by a factor 2 . The high pass or detail component characterizes the image's high frequency information with vertical orientation and the low pass approximation component contains its low frequency, vertical information. Both sub images are filtered column wise and down sampled to yield four quarter size output sub images. These sub images, $w_{\varphi}, w_{\psi}^{H}, w_{\psi}^{V}, w_{\psi}^{D}$ are the inner products of $\mathrm{f}(\mathrm{x}, \mathrm{y})$ and the two dimensional scaling and wavelet functions followed by down sampling by two in each dimension.

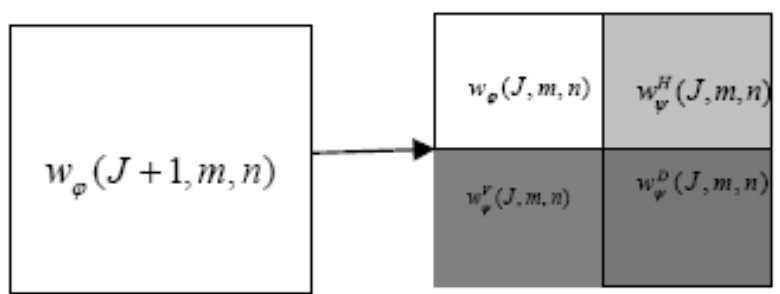

Fig 4: Single level DWT Decompositions

\subsubsection{Discrete Cosine transform}

DCT is [8-10] a well-known signal analysis tool used in compression due to its compact representation power. It's known, that Karhunen-Loeve transform (KLT) is the optimal transform in terms of information packing, however, its data dependent nature makes it infeasible to implement in some practical tasks. Moreover, DCT closely approximates the compact representation ability of the KLT, which makes it a very useful tool for signal representation both in terms of information packing and in terms of computational complexity due to its data independent nature.

DCT helps in separating the image into parts (or spectral sub-bands) of differing importance (with respect to the image's visual quality). The general equation for a 1D ( $N$ data items) DCT is defined by the following equation:

$$
F(u)=\sqrt{\left(\frac{2}{N}\right)} \sum_{i=0}^{N-1} A(i) * \cos \left(\frac{u(2 i+1) \pi}{2 N}\right) * f(i)
$$

where, $f(i)$ is the input sequence and

$$
A(i)=\frac{1}{\sqrt{2}} \text { for } \mathrm{u}=0 \text { and it is } 0 \text { otherwise }
$$

\subsubsection{Discrete Sine Transform (DST)}

The DST is a frequency transform similar to the discrete Fourier transform (DFT), but using only real numbers [10]. It is equivalent to the imaginary parts of a DFT of roughly twice the length, operating on real data with odd symmetry. The general equation for a 1D ( $N$ data items) DST is defined by the following equation:

$$
F(u)=\sqrt{\frac{2}{N+1}} \sum_{i=0}^{N-1} \sin \left(\frac{\pi(i+1)(U+1)}{N+1}\right) * f(i)
$$

where $f(i)$ is the input sequence.

\section{IMPLEMETATION}

The implementation is divided into three parts known as testing for accuracy, testing for authentication and offline applications.

\subsection{Accuracy}

We have collected 5 signature samples from 75 persons out of which we have considered three signatures samples for database and two for testing the accuracy. In testing phase, when the query signature is entered, the feature vector corresponding to this signature is calculated and it is compared with the feature vectors of each of the database signatures. For comparison, we use Euclidean distance model which calculates the Euclidean distance between feature 
vectors of the test signature and feature vectors of the database signatures [11]. To find out the true acceptance rate (TAR) we have used the following formula

$$
T A R=\frac{\text { No of accepted true signatures }}{\text { total no. of tested signatures }}
$$

First we have tested the accuracy of the system by considering the only DWT approximate coefficients under various levels and the results are tabulated in table 1 .

Table 1. TAR for DWT

\begin{tabular}{|c|c|c|}
\hline $\begin{array}{c}\text { No. of } \\
\text { levels }\end{array}$ & $\begin{array}{c}\text { No of } \\
\text { Coefficients }\end{array}$ & $\begin{array}{c}\text { TAR in } \\
\%\end{array}$ \\
\hline 1 & 8182 & 48 \\
\hline 2 & 2048 & 65 \\
\hline 3 & 512 & 89 \\
\hline 4 & 128 & 79 \\
\hline 5 & 32 & 74 \\
\hline 6 & 8 & 71 \\
\hline
\end{tabular}

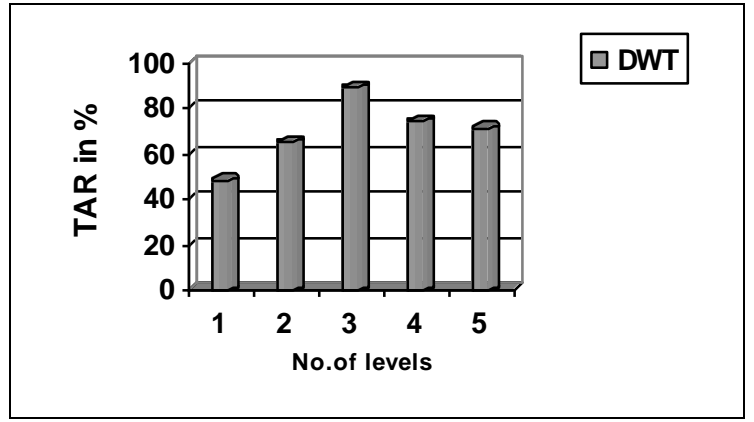
only

Fig 5: Results of Signature Recognition using DWT

From fig. 5, it is clear that, maximum recognition rate is possible with level 3 DWT. Hence for cascading the sinusoidal transforms, we have decided to use 3 levels DWT with 512 coefficients. Now the results of cascading operations are listed in table 2 .

Table 2. TAR for Cascading Operations

\begin{tabular}{|c|c|c|}
\hline $\begin{array}{c}\text { No of } \\
\text { Coefficients }\end{array}$ & $\begin{array}{c}\text { TAR in \% } \\
\text { DWT-DCT }\end{array}$ & $\begin{array}{c}\text { TAR in \% } \\
\text { DWT-DST }\end{array}$ \\
\hline 8 & 81 & 84 \\
\hline 16 & 89 & 94 \\
\hline 32 & 95 & 96 \\
\hline 128 & 94 & 95 \\
\hline 256 & 88 & 88 \\
\hline
\end{tabular}

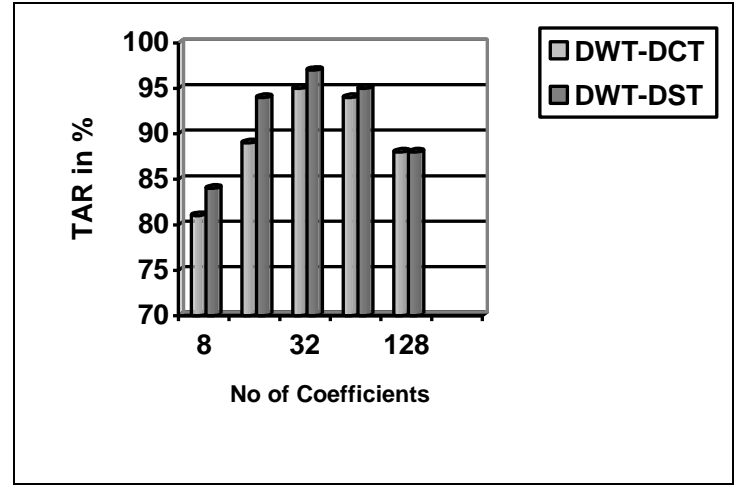

Fig 6: Results of Cascading Operations

From fig.6. , we can find that cascading the transforms results in better recognition rate.

\subsection{Testing for authentication}

\subsubsection{Need for image Registration}

The difficulties associated with signature verification systems due to the extensive intra-class variations, make signature verification a difficult pattern recognition problem. Examples of the various alterations observed in the signature of an individual have been illustrated in fig. 7 .

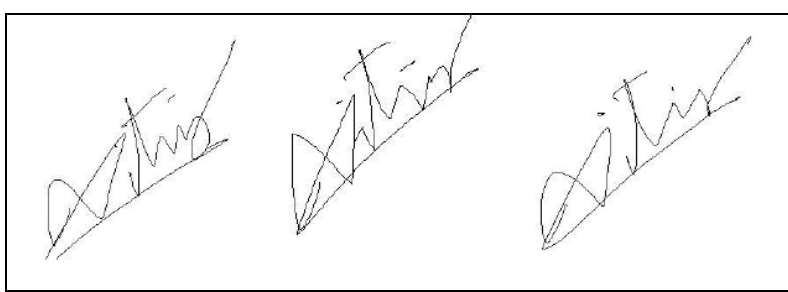

Fig 7: Variations in an Individual Signature

Hence, to reduce errors and the inefficiency problems associated with these systems, the intra-class variations in the signatures need to be minimized. This involves eliminating or reducing the rotation, scaling and translation factors between the reference and the test signature images [12-16]. Hence our system does not require multiple signature reference samples for training in order to achieve high levels of accuracy. The image registration has been implemented using the following three steps

i) Rotation Correction using Normalized Correlation: The following algorithm finds the correlation between original image and sensed images [12, 13]. It applies the transformation parameters on sensed images so that maximum correlation between original image and sensed images are achieved. If $A(m, n)$ is reference image and $B(m, n)$ is sensed image and then correlation coefficient between $A$ and $B$ is found by

$$
r=\frac{\sum_{m} \sum_{n}\left(A_{m n}-A_{0}\right)\left(B_{m n}-B_{0}\right)}{{\sqrt{\sum_{m} \sum_{n}\left(A_{m n}-A_{0}\right)^{2} \sum_{m} \sum_{n}\left(B_{m n}-B_{0}\right)}}^{2}}
$$


Where $r$ is correlation coefficient which value should be between 0 to 1 . Minimum value of $r$ shows the dissimilarity of image and for the same images it will have value $1 . \mathrm{A}_{0}$ and $\mathrm{B}_{0}$ represent mean of image $A$ and $B$ respectively. The following steps perform rotation correction.

- $\quad$ The user image and query image are resized to 128 $\mathrm{x} 256$, converted into gray scale image and then binarized.

- Both the signatures are cropped from background.

- Starting with the angle as $-60^{\circ}$, in increments of 0.5 $\circ$, normalized correlation values between preprocessed reference image and user image are calculated.

- The maximum cross-correlation value refers that the images are now registered and current angle of rotation represents the angle between the two images.

- Now the query image is rotated by the angle obtained.

ii) Scaling correction using Image Cropping: The size of the signature depends upon the space provided for signing. Thus, before extraction of feature points, it is essential that any scaling, if present in the query signature, be removed. Upon removal of background, the ratio of height between the query template and reference template gives vertical scaling and ratio of width gives horizontal scaling. Scaling ratio (SR) is calculated by $t$ equation 8 and the user image is resized as per the obtained scaling ratio

$$
S R=\frac{\text { Size of reference image }}{\text { Size of query image }}
$$

iii) Translation correction by background filling: On the signature tablet used for taking signature input, the user is free to sign without using any fixed starting point. This may introduce translation in horizontal and/or vertical direction. This problem is already overcome by cropping since cropping process truncates the extra background region. Thus, translation is removed completely. After correcting the angle of rotation, the user image pre-processed copy is cropped to eliminate translation and the image so obtained is a case of pure scaling. In fig 8, the first template is the reference image, second template is the query image and the third image is the registered query image.
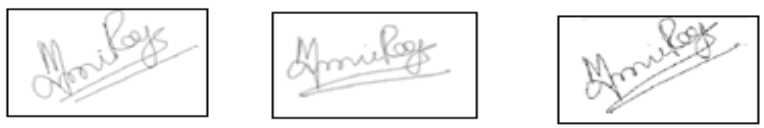

Fig 8: Image Registration

During the authentication phase, the query signature is collected from the user and after image registration with the reference image, the feature vectors are extracted from the query image and compared with data base feature vectors. If there is a match, the authentication is granted otherwise the user is denied from authentication.

\subsection{Offline Applications}

In off-line signature verification, the signature is available on a document which is scanned to obtain its digital image. In all applications where handwritten signatures currently serve as means of authentication, automatic signature verification can be used such as cashing a check, signing a credit card transaction or authenticating a legal document. Fig 9 shows the scanned copy of a bank cheque.

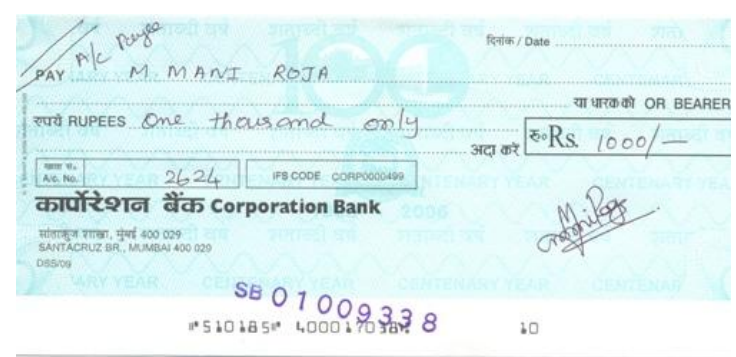

Fig 9: Scanned Cheque

To verify the signature in the cheque, with signature sample in the data base, we first cropped the signature from the cheque. During preprocessing, this colour sample is first converted into gray image then to binary image as shown in fig. 10 .

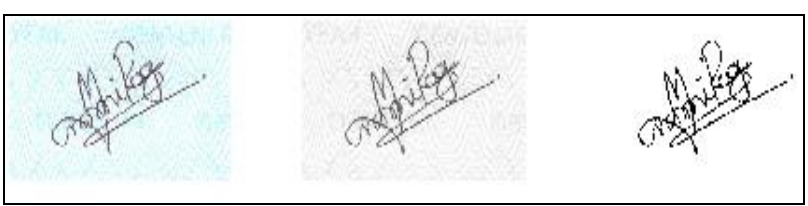

Fig 10: Preprocessing of extracted signature

After resizing, image registration is done with respect to the data base signature template. Now the template is ready for comparison with the reference signature. The feature vectors are extracted from the query image and the reference image and a match score is calculated using the Euclidean distance classifier. If the score is above the predefined threshold value, the signature is accepted for further transactions otherwise it is rejected.

\section{CONCLUSION}

The objective of this work was to investigate the cascaded transforms approach for signature recognition to achieve higher performance. Our results show that when DWT is used alone it give a true acceptance rate of $89 \%$ with level 3 DWT and when DWT is cascaded with DCT, we get an a true acceptance rate of $94 \%$ and when DWT is cascaded with DST , we get the rate as of $96 \%$. Since we have used image registration, multiple sample collection from the users has been reduced. With the Banking applications, we found out that the system works for online as well as offline applications. 


\section{REFERENCES}

[1] Anil K. Jain, Arun Ross and Salil Prabhakar, "An Introduction to Biometric Recognition", IEEE Transactions on Circuits and Systems for Video Technology, Special Issue on Image- and Video-Based Biometrics, Vol. 14, No. 1 , January 2004.

[2] Sulochana Sonkamble, Ravindra Thool , Balwant Sonkamble, "Survey of Biometric Recognition Systems and their applications", Journal of Theoretical and Applied Information Technology 2005 - 2010.

[3] Payman Moallem1, Seyed Amirhassan Monadjemi,

"Dynamic Online Signatures Recognition using a novel Signature base normalized feature string and MLP Neural Network" , Iranian Journal of Engineering sciences , Vol.1, No.1,2007.

[4] Lin Hong and Anil Jain, "Integrating Faces and Fingerprints

for Personal Identification", IEEE Transactions on Pattern Analysis and Machine Intelligence", Vol.20, No.12, December 1998.

[5] Arun Ross, Anil Jain, "Information fusion in biometrics", Pattern Recognition Letters 24 (2003) 2115-2125.

[6] Ning - Ning Liu, Yun-Hong Wang, "Fusion of Global and Local information for an online Signature Verification system", Proceedings of the Seventh International Conference on Machine Learning and Cybernetics, Kunming, 12-15 July 2008.

[7] WACOM Bamboo Digital Pen Tablet, www.wacom.co.in /bamboo, June 2011.

[8] S. Jayaraman, S Esakkirajan, T Veerakumar, "Digital Image Processing”, by Mc Graw Hill, 2008.
[9] Rafael C. Gonzalez, Richard E. Woods, 'Digital Image Processing', 9th Edition, Prentice Hall, New York, Sept 09.

[10] Vaishali Kulkarni, H. B. Kekre, "Comparative Analysis of Automatic Speaker Recognition using Kekre's Fast Codebook GeneratioAlgorithm in Time and Transform Domain", International Journal of Computer Applications Volume 7- No.1, September 2010 pp-37-41.

[11]Xin Pan , Qiuqi Ruan1 , Yanxia Wang, "Palm print Recognition using Fusion of Local and Global features", Proceedings of 2007 International Symposium on Intelligent Signal Processing and Communication Systems Nov.28Dec.1, 2007.

[12] Aman Chadha, Divya Jyoti, M. Mani Roja, "Rotation, Scaling and Translation Analysis of Biometric Signature Templates", Int. J. Comp. Tech. Appl., Vol 2 (5), 14191425 , Sept 2011

[13]Mehfuza Holia, V.K Thakur, “ Image Registration for recovering Affine Transformation using Nelder Mead Simplex method for optimization", International Journal of Image Processing (IJIP) Volume(3), Issue(5).

[14] G. Wolberg and S. Zokai, "Robust Image Registration Using Log-Polar Transform", Proceedings of the IEEE International Conference on Image Processing, Sep. 2000.

[15] Z. Y. Cohen, "Image registration and object recognition using affine invariants and convex hulls", IEEE Transactions on Image Processing, July 1999, pp. 1-3.

[16] N. Chumchob and K. Chen, "A Robust Affine Image Registration Method", International Journal of Numerical Analysis and Modeling, Volume 6, Number 2,2009, pp. 311-334. 